\title{
OPTIMAL DETECTION OF QAM IN A MAN-MADE NOISE ENVIRONMENT
}

\author{
Mustapha Hamza, Huu Tuê Huynh, Paul Fortier \\ Department of Electrical and Computer Engineering \\ Laval University, Ste-Foy, Quebec Canada G1K 7P4 \\ PH: 418-565-3555 FAX: 418-656-3159 \\ e-mail: \{mhamza, huynh, fortier $\} @$ gel.ulaval.ca
}

\begin{abstract}
It is known that in the personal communication systems environment, additive noise is not Gaussian. Experimental studies show that this type of noise obeys the class A Middleton man-made noise statistics. The influence of such a noise is very severe on the probability error rate for a matched filter detector (1). To improve the detection performance, a non-linear detector should be used. This structure is based on the maximum likelihood approach which exploits multiple samples of the incoming signal. The improvement has been established by Spaulding and Middleton for the binary case (2). This paper generalizes this approach to $M$-ary signal detection, namely $M$-QAM, which is the most popular scheme in practice.
\end{abstract}

\section{INTRODUCTION}

Digital communication systems are being used more and more in consumer products, such as Personal Communication Systems (PCS). Their performance is generally defined by the symbol or bit error rate in the presence of additive white Gaussian noise. It is well known that the household environment is subjected to man-made noise (e.g. microwave ovens, computers, television receivers,...). Experimentally, the observed man-made noise appears as large impulses occurring during the detection interval (3), (4). In a series of papers by the authors, it was shown that this type of non-Gaussian noise has a dramatic effect on the performance of QAM modems when linear (matched fitter) detection is used (5), (6), (7). The performance is shown to be strongly dependent on two parameters: the impulsive index $(A)$ and the power ratio of the impulsive noise component and the Gaussian noise component $\left(\beta=\sigma_{G}^{2} / \sigma_{I}^{2}\right)$. Recently, the multicarrier modulation (MCM) transmission scheme was shown to be effective in combatting this type of noise by increasing the impulsive index of the noise (7). However, the overall performance of MCM in nonGaussian noise can be improved if optimal detection is used on each of the sub-carriers.
Spaulding and Middleton (2) proposed the use of multiple samples of received symbols, then applied the maximum likelihood test to construct the optimum receiver. For $M$-QAM detection, this approach can be used to improve the receiver performance. This paper presents bounds for the symbol error rate of the optimum passband and baseband receivers.

\section{NOISE MODEL AND MATCHED FILTER RECEIVER}

In this work, the man-made noise is modeled using Middleton class A non-Gaussian noise statistics (8). Let $N(t)$ be the noise at the output of the narrowband IF stage. It can be expressed as

$$
\begin{aligned}
N(t) & =X(t) \cos \left(\omega_{0} t\right)+Y(t) \sin \left(\omega_{0} t\right) \\
& =\rho(t) \cos \left(\omega_{0} t+\phi(t)\right)
\end{aligned}
$$

The Middleton class A statistics of the noise envelope $\rho(t)$ is given by

$$
\begin{gathered}
f_{P}(\rho)=\exp (-A) \sum_{k=0}^{\infty} \frac{A^{k} \rho}{k ! \sigma_{k}} \exp \left(-\frac{\rho^{2}}{2 \sigma_{k}^{2}}\right) \\
\sigma_{k}^{2}=\frac{(k / A)+\beta}{1+\beta}
\end{gathered}
$$

As suggested by the CCITR (4), the phase of non-Gaussian noise is assumed uniformly distributed over the interval $[0,2 \pi]$ and statistically independent of its envelope. Using this assumption, the joint probability density function of the in-phase and quadrature noise components at the output of the matched filter receiver is given by

$$
f_{X, Y}(x, y)=\exp (-A) \sum_{k=0}^{\infty} \frac{A^{k}}{k !} \frac{1}{2 \pi \sigma_{k}^{2}} \exp \left(-\frac{x^{2}+y^{2}}{2 \sigma_{k}^{2}}\right)
$$


where $A$ and $\beta$ are respectively defined as the impulsive index and the power of the Gaussian noise component over the impulsive noise component.

Let $\mathbf{S}_{m}$ be the complex transmitted symbol. The baseband received data is then

$$
R=S+N
$$

where $\boldsymbol{S}=C_{x}+j C_{y}$ and $\boldsymbol{N}=X+j Y$; the subscript $m$, being irrelevant, has been deleted. The vector $\left(C_{x}, C_{y}\right)$ corresponds to a point in the $M$-QAM constellation, associated with the transmitted symbol. The decision device detects $\left(C_{x}, C_{y}\right)$ based on the complex observation $\boldsymbol{R}$.

Using the analytical expression of the pdf, the symbol error rate of the matched filter receiver has been shown to be (1)

$$
\begin{aligned}
& P(\varepsilon)= \\
& 4 \exp (-A) \sum_{k=0}^{\infty} \frac{A^{k}}{k !}\left(1-\frac{1}{\sqrt{M}}\right) P_{k}\left[1-\left(1-\frac{1}{\sqrt{M}}\right) P_{k}\right]
\end{aligned}
$$

where $P_{k}$ is given by

$$
P_{k}=\frac{1}{2} \operatorname{erfc}\left[\sqrt{\frac{3}{2(M-1)} \frac{E_{a}}{2 \sigma_{k}^{2}}}\right]
$$

$E_{a}$ is the mean energy of each signal constellation.

\section{OPTIMUM COHERENT RECEIVER}

Following the approach proposed by Spaulding and Middleton (2), the received signal vector is composed of $E$ samples of the incoming signal. With these samples, it is well known that the optimum structure uses a Bayesian strategy to choose the most probable signal over $M$ hypotheses. Hence, the optimum receiver computes $M$ likelihood functions based on the received signal vector and decides that the transmitted signal corresponds to the maximum of these functions. In this paper, this approach is extended to the $M$-QAM signal set in the IF domain as well as in baseband detection.

\section{Optimum Passband Detection}

Let $\left\{S_{i j}=C_{i}+j C_{j}\right\}$ be the complex symbol set corresponding to an $M$-QAM constellation and let $S_{(i, j)}(t)$ be the transmitted signal. It is then defined as
$S_{(i, j)}(t)=\operatorname{Re}\left\{S_{i j} e^{j \omega_{o} t}\right\}=C_{i} \cos \left(\omega_{o} t\right)-C_{j} \sin \left(\omega_{o} t\right)(7)$

The received signal is then $R(t)=S(t)+N(t)$, where $N(t)$ is the class A Middleton non-Gaussian noise. For passband detection, the received signal is sampled at the instants $t_{e}$ to give $E$ samples $R\left(t_{e}\right)$ :

$$
\begin{gathered}
R\left(t_{e}\right)=S_{(i, j)}\left(t_{e}\right)+N\left(t_{e}\right) \\
t_{e}=\frac{T}{E-1}(e-1) \quad e=1,2, \ldots, E
\end{gathered}
$$

where $T$ is the symbol duration. The sample $S_{(i, j)}\left(t_{e}\right)$, noted as $S_{e(i, j)}$, is given by

$$
\begin{aligned}
& S_{(i, j)}\left(t_{e}\right)=S_{e(i, j)}= \\
& C_{i} \cos \left(\left(\frac{Q}{E-1}(e-1)\right)-C_{j} \sin \left(\frac{Q}{E-1}(e-1)\right)\right)
\end{aligned}
$$

with $Q=\omega_{0} T$.

The optimum receiver computes $M$ likelihood functions

$$
L_{(i, j)}=\prod_{e=1}^{E}\left[\sum_{k=0}^{\infty}\left[\frac{A^{k}}{k ! \sigma_{k}} \exp \left(-\frac{\left(r_{e}-S_{e(i, j)}\right)^{2}}{2 \sigma_{k}^{2}}\right)\right]\right]
$$

The vector index $(i, j)$ corresponds to a point of the $M$ QAM constellation. The optimum Bayesian decision is then given by

$$
S_{(\hat{i}, \hat{j})}=\arg \left\{\max _{i, j}\left(L_{(i, j)}\right)\right\}
$$

The computation of the error rate for this optimum receiver is mathematically intractable. However, it can be upper bounded using the union bound technique and the explicit probability error of the two simple hypothesis $\left(H_{1}\right.$ and $\left.H_{2}\right)$ situation. In the following, the first main task is to compute the error probability to discriminate between these two hypotheses

$$
\begin{array}{lll}
H_{1}: & R_{\left(i_{1}, j_{1}\right)}(t)=S_{\left(i_{1}, j_{1}\right)}(t)+N(t) & 0 \leq t<T \\
H_{2}: & R_{\left(i_{2}, j_{2}\right)}(t)=S_{\left(i_{2}, j_{2}\right)}(t)+N(t) & 0 \leq t<T
\end{array}
$$

The $E$ samples of the received signal can be represented in vector form as follows:

$$
\boldsymbol{R}=\boldsymbol{S}+\boldsymbol{N}
$$


where $\boldsymbol{R}$ is a vector of $E$ components, each of which corresponds to the to $e^{\text {th }}$ received sample of $R(t)$ (despite their different mathematical meanings, we use the same symbols for both equations (14) and (4); there is no confusion because they have the same physical meaning for different contexts). Assuming the samples of the noise are identically distributed and independent, the likelihood ratio is defined as

$$
\begin{aligned}
& \Lambda_{\left(i_{1}, j_{1}\right)\left(i_{2}, j_{2}\right)}= \\
& \prod_{e=1}^{E}\left[\sum_{k=0}^{\infty}\left[\frac{A^{k}}{k ! \sigma_{k}} \exp \left(-\frac{\left(r_{e}-S_{e\left(i_{1}, j_{1}\right)}\right)^{2}}{2 \sigma_{k}^{2}}\right)\right]\right] \\
& \prod_{2} \\
& \prod_{e=1}^{E}\left[\sum_{k=0}^{\infty}\left[\frac{A^{k}}{k ! \sigma_{k}} \exp \left(-\frac{\left(r_{e}-S_{e\left(i_{2}, j_{2}\right)}\right)^{2}}{2 \sigma_{k}^{2}}\right)\right]\right]
\end{aligned}
$$

where $r_{e}$ is the $e^{\text {th }}$ received signal sample. The binary situation error rate is given by

$$
\begin{array}{r}
\left(P_{e}\right)_{\left(i_{1}, j_{1}\right)\left(i_{2}, j_{2}\right)}=\int_{-\infty}^{\infty} \min \left\{\frac{1}{M} f\left(r_{e} \mid H_{\left(i_{1}, j_{1}\right)}\right),\right. \\
\left.\frac{1}{M} f\left(r_{e} \mid H_{\left(i_{2}, j_{2}\right)}\right)\right\} d r_{e}
\end{array}
$$

Given that

$$
\min \{a, b\} \leq a^{\alpha} b^{1-\alpha}
$$

for all $0 \leq \alpha \leq 1, a \geq 0, b \geq 0$, it can be shown that an upper bound of (16) is given by

$$
\begin{aligned}
& \left(P_{e}\right)_{\left(i_{1}, j_{1}\right)\left(i_{2}, j_{2}\right)} \leq \\
& \frac{1}{M} \int_{-\infty}^{\infty} f\left(r_{e} \mid H_{\left(i_{1}, j_{1}\right)}\right)^{\alpha} f\left(r_{e} \mid H_{\left(i_{2}, j_{2}\right)}\right)^{1-\alpha} d r_{e}
\end{aligned}
$$

Let us define

$$
\begin{gathered}
\rho_{e\left(i_{1}, j_{1}\right)\left(i_{2}, j_{2}\right)}=\frac{S_{e\left(i_{1}, j_{1}\right)}-S_{e\left(i_{2}, j_{2}\right)}}{2} \\
x=r_{e}-\frac{S_{e\left(i_{1}, j_{1}\right)}+S_{e\left(i_{2}, j_{2}\right)}}{2}
\end{gathered}
$$

Then

$$
\left(P_{e}\right)_{\left(i_{1}, j_{1}\right)\left(i_{2}, j_{2}\right)} \leq \frac{1}{M} \prod_{e=1}^{E} I_{\alpha}\left(\rho_{e\left(i_{1}, j_{1}\right)\left(i_{2}, j_{2}\right)}\right)
$$

where

$$
\begin{aligned}
& I_{\alpha}\left(\rho_{e\left(i_{1}, j_{1}\right)\left(i_{2}, j_{2}\right)}\right)= \\
& \int_{-\infty}^{\infty}\left(\left[\sum_{k=0}^{\infty}\left[\frac{A^{k}}{k !} \frac{1}{\sqrt{2 \pi \sigma_{k}^{2}}} \exp \left(-\frac{\left(x-\rho_{e\left(i_{1}, j_{1}\right)\left(i_{2}, j_{2}\right)}\right)^{2}}{2 \sigma_{k}^{2}}\right)\right]\right)^{\alpha} \times\right. \\
& \left(\left[\sum_{k=0}^{\infty}\left[\frac{A^{k}}{k !} \frac{1}{\sqrt{2 \pi \sigma_{k}^{2}}} \exp \left(-\frac{\left(x+\rho_{e\left(i_{1}, j_{1}\right)\left(i_{2}, j_{2}\right)}\right)^{2}}{2 \sigma_{k}^{2}}\right)\right]\right)^{1-\alpha} d x\right.
\end{aligned}
$$

Since this function is symmetrical, it can be shown that the tightest bound in regards of $\alpha$ occurs at $\alpha=1 / 2$. Hence

$$
\left(P_{e}\right)_{\left(i_{1}, j_{1}\right)\left(i_{2}, j_{2}\right)} \leq \frac{1}{M} \prod_{e=1}^{E} I_{\frac{1}{2}}\left(\rho_{e\left(i_{1}, j_{1}\right)\left(i_{2}, j_{2}\right)}\right)
$$

An upper bound of the total symbol error rate, using the union bound technique, is then given by

$$
P(\varepsilon) \leq \frac{2}{M^{2}(M-1)}\left[\sum_{\substack{\left(i_{1}, j_{1}\right) \\\left(i_{2}, j_{2}\right)}} \prod^{E=1} I_{\frac{1}{2}}\left(\rho_{e\left(i_{1}, j_{1}\right)\left(i_{2}, j_{2}\right)}\right)\right]
$$

This bound is clearly defined by the parameters $\rho_{e\left(i_{1}, j_{1}\right)\left(i_{2}, j_{2}\right)}$ which are the distances between the $e^{\text {th }}$ samples of the two signals $S_{\left(i_{1}, j_{1}\right)}(t)$ and $S_{\left(i_{2}, j_{2}\right)}(t)$.

\section{Baseband detection}

In baseband detection, the in-phase and quadrature components are sampled and separately processed using the same approach as in the IF case (see Figure 1). The total error rate is bounded by twice the error rate for one of the components for which the signal space dimension is reduced from $M$ to $K=\sqrt{M}$.

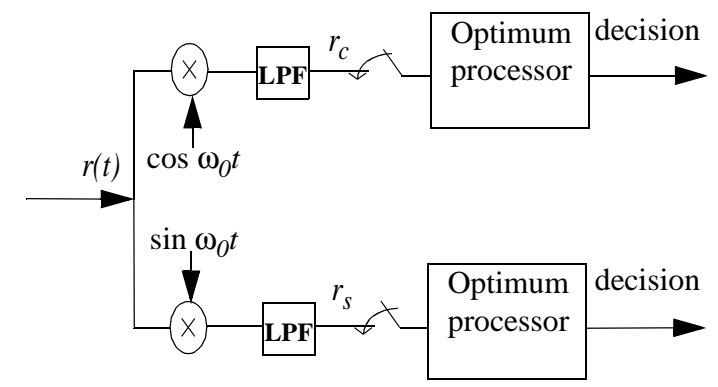

Figure 1: Optimum baseband detector 
Using the same arguments as in the preceding section, an upper bound can be evaluated as

$$
P(\varepsilon) \leq \frac{4}{K^{2}(K-1)}\left[\sum_{i_{1}=1}^{K-1} \sum_{i_{2}=i_{1}+1}^{K} \prod_{e=1}^{E} I_{\frac{1}{2}}\left(\rho_{e\left(i_{1}, i_{2}\right)}\right)\right]
$$

with

$$
\begin{aligned}
& I_{\frac{1}{2}}\left(\rho_{e\left(i_{1}, i_{2}\right)}\right)= \\
& \int_{-\infty}^{\infty}\left(\left[\sum_{k=0}^{\infty}\left[\frac{A^{k}}{k !} \frac{1}{\sqrt{2 \pi \sigma_{k}^{2}}} \exp \left(-\frac{\left(x-\rho_{e\left(i_{1}, i_{2}\right)}\right)^{2}}{2 \sigma_{k}^{2}}\right)\right]\right]\right)^{\frac{1}{2}} \times \\
& \left(\left[\sum_{k=0}^{\infty}\left[\frac{A^{k}}{k !} \frac{1}{\sqrt{2 \pi \sigma_{k}^{2}}} \exp \left(-\frac{\left(x+\rho_{e\left(i_{1}, i_{2}\right)}\right)^{2}}{2 \sigma_{k}^{2}}\right)\right]\right]\right)^{\frac{1}{2}} d x
\end{aligned}
$$

where

$$
\rho_{e\left(i_{1}, i_{2}\right)}=\frac{C_{i_{1}}-C_{i_{2}}}{2} \quad \text { and } \quad x=r_{e}-\frac{C_{i_{1}}+C_{i_{2}}}{2}
$$

As before, the upper bound of the error rate is perfectly defined by $\rho_{e\left(i_{1}, i_{2}\right)}$, the distances between the $e^{\text {th }}$ samples of the in-phase (or quadrature) components $C_{i_{1}}(t)$ and $C_{i_{2}}(t)$.

\section{TYPICAL RESULTS AND DISCUS- SION}

Some typical results of the linear receiver performance are shown in Figure 2 and Figure 3. We observe that when the noise is very impulsive (low impulsive index $A$ and small $\beta$ ), the BER exhibits a threshold phenomenon, represented by a plateau on the BER curves. As the noise approaches the Gaussian case (relatively high impulsive index or very high $\beta$ ), the BER curves approach the Gaussian performance, which is quite natural.

For the optimum detection, we use the numerical values $E=5,10$ and $Q=100$, which are the same as used in (2). Figures 4 and 5 show the SER performance for IF detection. Generally, the optimum detector offers a much better performance compared to the linear one. This enhancement can be mainly explained by the fact that the detection is optimized with the use of $E$ available signal samples. Furthermore, we can see intuitively that using $E$ samples improves the signal to noise ratio. Due to this fact, the SER performance must be enhanced when $E$ is increased. This phenomenon is clearly observed in fig-

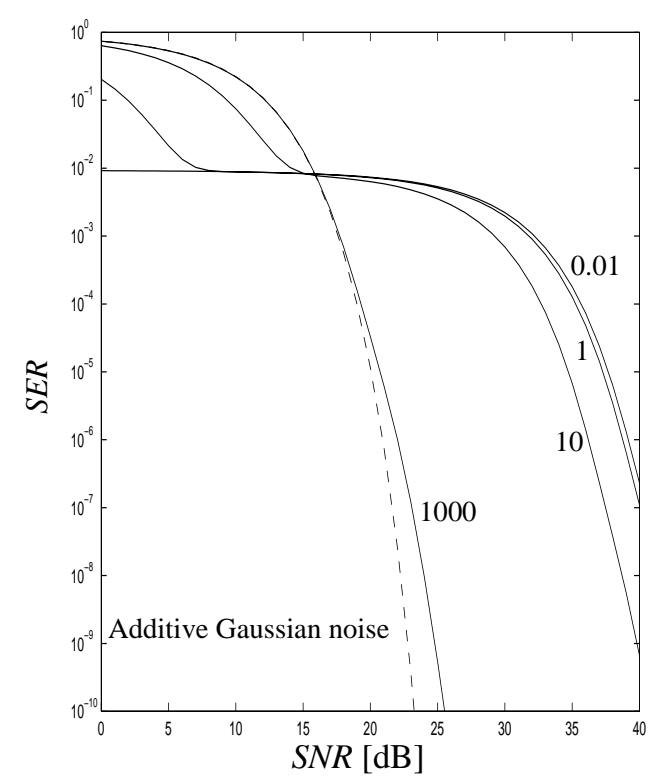

Figure 2: SER vs. SNR for matched filter receiver $(M F)$ with various values of $\beta, M=16$ and $A=0.01$

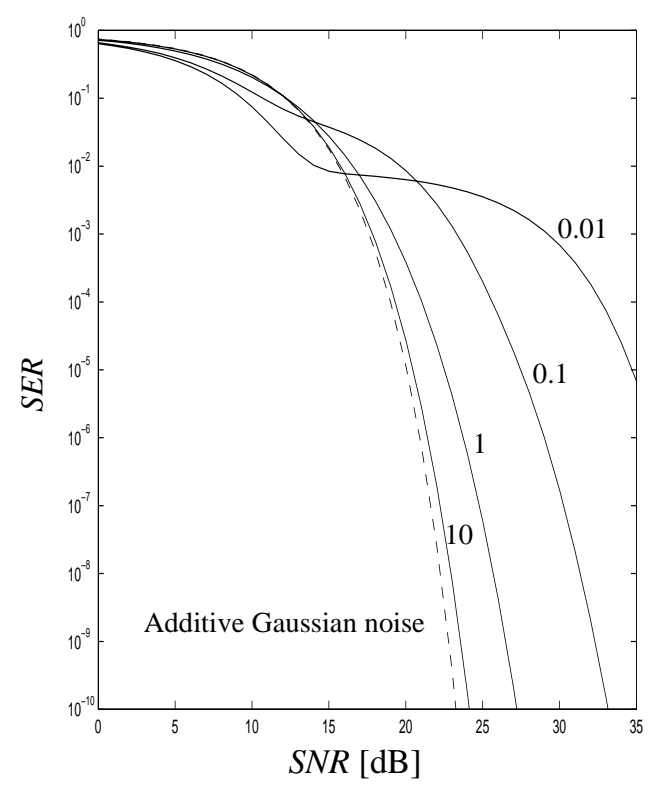

Figure 3: SER vs. SNR for matched filter receiver $(M F)$ with various values of $A, M=16$ and $\beta=1$

ures 4 and 5. When the noise is relatively impulsive (small $A$ and small $\beta$ ), the enhancement in SER is remarkable (see Figure 4). When the noise becomes more Gaussian (relatively high $A$ or very high $\beta$ ), the optimum receiver offers less improvement in SER performance, compared to the linear receiver (see Figure 5). In Figure 5 , we observe another interesting phenomenon, that for 


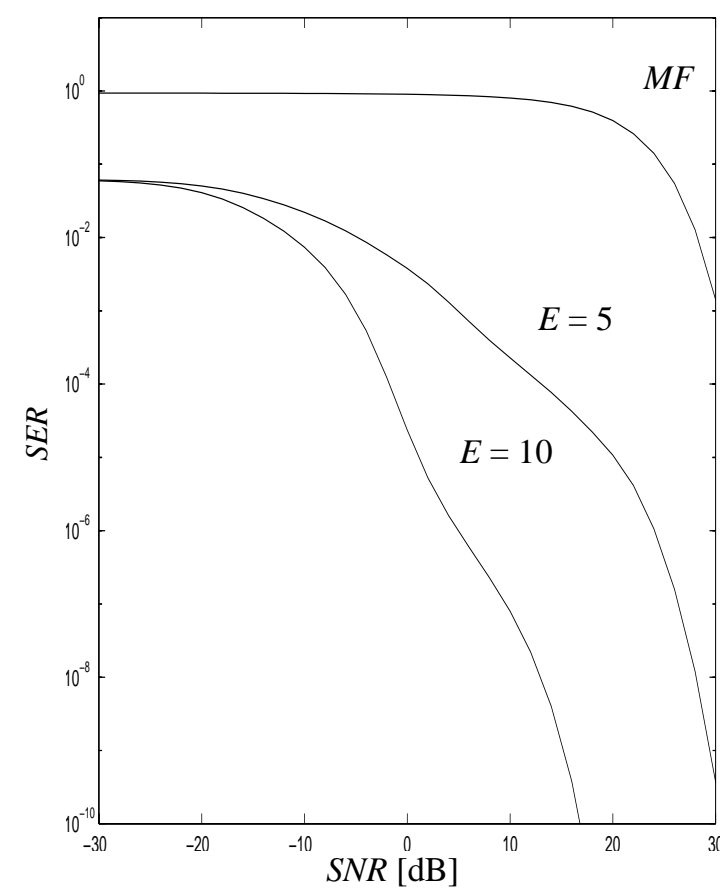

Figure 4: $S E R$ vs. SNR for optimum passband detection for $M=16$ with $A=0.1$ and $\beta=0.1$

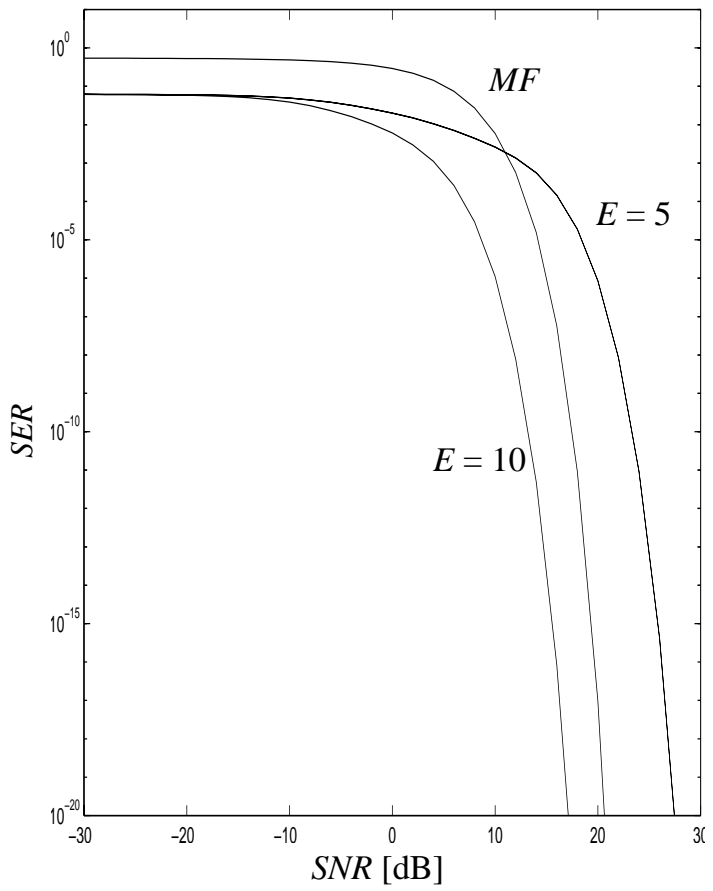

Figure 5: SER vs. SNR for optimum passband detection for $M=16$ with $A=10$ and $\beta=0.1$

high SNR, the linear receiver might offer a better performance when the number of samples used by the optimum receiver is not high enough. This apparent contradiction can be explained as follows. The linear receiver, when the noise is purely Gaussian, offers the minimum SER,

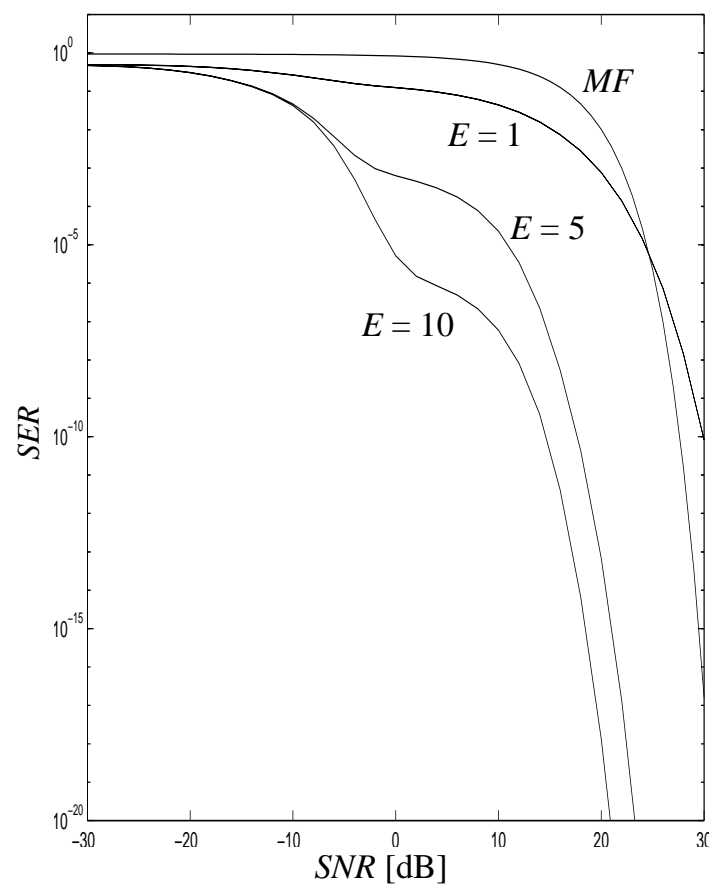

Figure 6: SER vs. SNR for optimum baseband detection for $M=16$ with $A=0.1$ and $\beta=0.1$

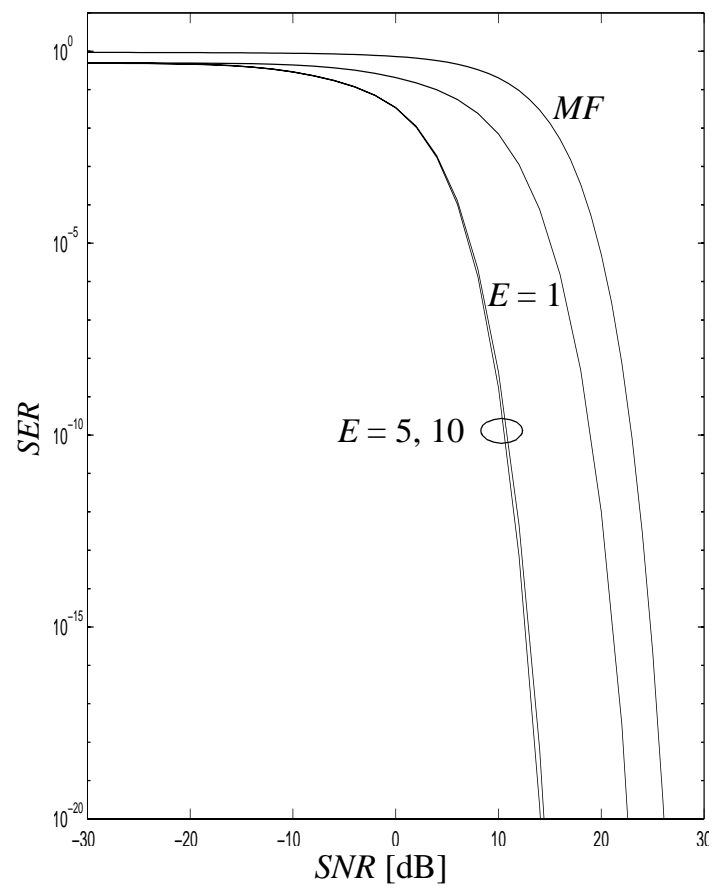

Figure 7: SER vs. SNR for optimum baseband detection for $M=16$ with $A=10$ and $\beta=0.1$

which corresponds at the same time to the maximum value of SNR at the output of the optimum linear receiver. When the noise approaches a Gaussian distribution, averaging over a high number of received signal samples might help to reach the SNR maximum value of the 
Gaussian case. When the number of available samples is not sufficient, the optimum SNR cannot be reached; as the noise is quasi-Gaussian, the correlator receiver has a better chance of offering an SER lower than the one provided by the non-linear processor using a small number of samples. This phenomenon is shown in Figure 5 for the case of $E=5$.

Figures 6 and 7 show the SER performances of the baseband optimum receiver. It is quite natural that the same behavior as in the IF case is observed for the baseband optimum detection. Furthermore, it is expected that the optimum baseband receiver offers a better SER performance than the IF optimum detector. This expectation is due to the fact that the overall processing considers samples of the received signal as dimensional vectors, whose components are separately processed. This comparison is shown on Figure 8, where we clearly observe that when the noise is relatively impulsive, the optimum baseband receiver reaches a much better performance compared to the other receivers.

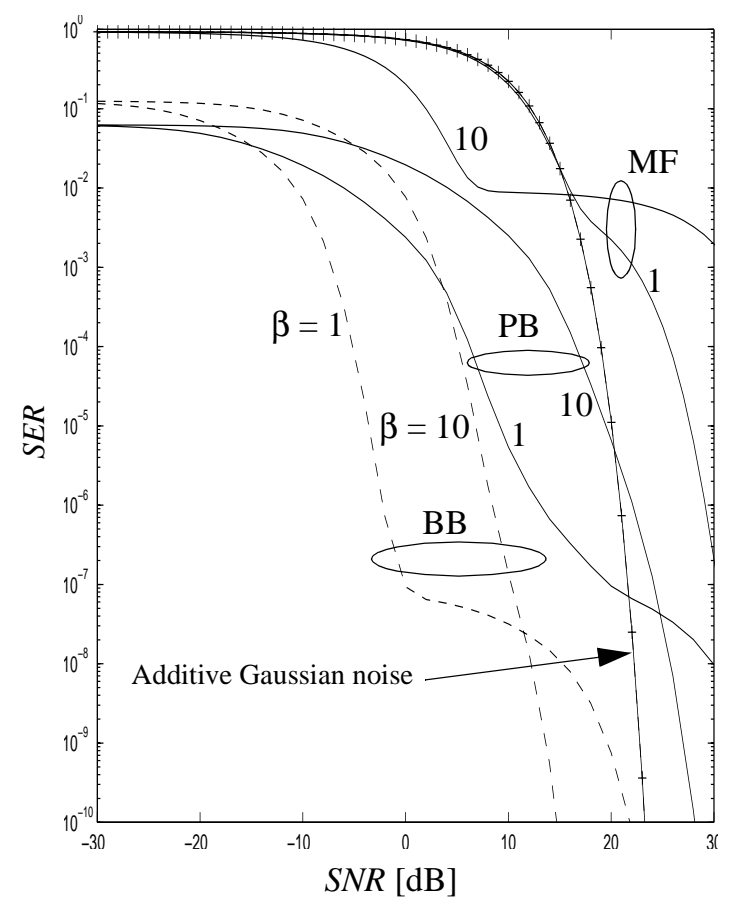

Figure 8: Comparison of optimum passband $(P B)$ and baseband $(B B)$ detection for $M=16$ with $A=0.01$

\section{REFERENCES}

(1) H. T. Huynh, B. Vo, P. Fortier, "Performance of MQAM in a Non-Gaussian Environment", International Journal of Electronics and Communications, vol. 51, no. 5, pp. 255-262, September 1997.

(2) A. R. Spaulding, D. Middleton, “Optimum Reception in an Impulsive Interference Environment - Part I: Coherent Detection", IEEE Transactions on Communications, vol. COM-25, no. 9, pp. 910-23, September 1977.

(3) N. Morinaga, "Research activities on radio communications in Osaka University", First Kansaï-Québec Workshop on Wireless Personal Communications, Montreal, October 18-19, 1994.

(4) E. N. Skomal, Man-Made Radio Noise, Van Nostrand Reinhold Company, New York, 1978.

(5) H. T. Huynh, P. Fortier, B. Vo, "Influence of Impulse Rate on the QAM Error Probability in a Non-Gaussian Noise Environment", Sixth Communications Theory Mini-Conference (Globecom'97), Phoenix, Arizona, November 5-7, 1997.

(6) H. T. Huynh, P. Fortier, "Influence of phase error on M-QAM demodulation in a non-Gaussian environment", 1998 Vehicular Technology Conference, Ottawa, May 18-21, 1998.

(7) H. T. Huynh, P. Fortier, G.-Y. Delisle, "Influence of a Class of Man-Made Noise on QAM Multicarrier Systems", Seventh Communication Theory Mini-Conference (Globecom'98), Sydney, Australia, 8-12 November, 1998.

(8) D. Middleton, "Statistical-physical models of electromagnetic interference", IEEE Trans. Electromag. Compat., vol. EMC-19, August 1977. 